\title{
Optimal Control of DC-DC Buck Converter via Linear Systems With Inaccessible Markovian Jumping Modes
}

\author{
Alessandro N. Vargas, Leonardo P. Sampaio, Leonardo Acho, Lixian Zhang, and João B. R. do Val
}

\begin{abstract}
The note presents an algorithm for the average cost control problem of continuous-time Markov jump linear systems. The controller assumes a linear state-feedback form and the corresponding control gain does not depend on the Markov chain. In this scenario, the control problem is that of minimizing the long-run average cost. As an attempt to solve the problem, we derive a global convergent algorithm that generates a gain satisfying necessary optimality conditions. Our algorithm has practical implications, as illustrated by the experiments that were carried out to control an electronic dc-dc buck converter. The buck converter supplied a load that suffered abrupt changes driven by a homogeneous Markov chain. Besides, the source of the buck converter also suffered abrupt Markov-driven changes. The experimental results support the usefulness of our algorithm.
\end{abstract}

Index Terms-DC-DC power converters, Markov processes, optimal control, optimization methods, stochastic processes.

\section{INTRODUCTION}

$\mathbf{M}$ ARKOV jump linear systems (MJLSs) have attracted widespread interest due to their appropriateness of representing processes subject to abrupt changes (see, for instance, the monograph [1]-[15] for a recent account). Applications of MJLS can be found in robotics [5], dc motors [16], [17], economy [18], and networks [19], [20], among others. In fact, the literature emphasizes the potential benefits of MJLS for real-time applications, and this brief provides a contribution toward this direction, as detailed next.

To clarify our contribution, consider the continuous-time MJLS defined on a filtered probability space $\left(\Omega, \mathcal{F}, \mathcal{F}_{t}, P\right)$ as follows [1]:

$$
\begin{array}{r}
d x(t)=A_{\theta(t)} x(t) d t+B_{\theta(t)} u(t) d t+H_{\theta(t)} d w(t) \\
\forall t \geq 0, \quad \theta(0)=\theta_{0}, \quad x_{0} \in \mathbb{R}^{r}
\end{array}
$$

Manuscript received October 6, 2015; accepted November 27, 2015. Manuscript received in final form December 7, 2015. This work was supported in part by the Brazilian Agencies Coordenação de Aperfeiçoamento de Pessoal de Nível Superior and Conselho Nacional de Desenvolvimento Científico e Tecnológico under Grant 471557/2009-9, Grant 304538/2009-4, and Grant 479673/2011-0, and in part by the Spanish Ministry of Economy and Competitiveness under Project DPI2012-32375/FEDER. Recommended by Associate Editor C. Prieur.

A. N. Vargas and L. P. Sampaio are with the Universidade Tecnológica Federal do Paraná, Cornélio Procópio 86300-000, Brazil (e-mail: avargas@utfpr.edu.br).

L. Acho is with the Control Dynamics and Application Laboratory, Departament de Matemàtica Aplicada III, Escola Universitària d'Enginyeria Tècnica Industrial de Barcelona, Universitat Politècnica de Catalunya, Barcelona 08036, Spain (e-mail: leonardo.acho@upc.edu).

L. Zhang is with the Space Control and Inertial Technology Research Center, Harbin Institute of Technology, Harbin 150001, China (e-mail: lixianzhang@hit.edu.cn).

J. B. R. do Val is with the School of Electrical and Computer Engineering, University of Campinas, Campinas-SP 13083-852, Brazil (e-mail: jbosco@dt.fee.unicamp.br).

Color versions of one or more of the figures in this paper are available online at http://ieeexplore.iee.org.

Digital Object Identifier 10.1109/TCST.2015.2508959 where $\{x(t)\}$ on $\mathbb{R}^{r},\{u(t)\}$ on $\mathbb{R}^{s}$, and $\{w(t)\}$ on $\mathbb{R}^{m}$ represent the continuous system state, control, and standard Brownian motion, respectively. The continuous-time homogeneous Markov chain $\{\theta(t)\}$ takes values in a finite set $\mathscr{S}:=\{1, \ldots, \sigma\}$ and has $\Lambda=\left[\lambda_{i j}\right], i, j=1, \ldots, \sigma$, as the stochastic rate matrix, which satisfies $\lambda_{i j} \geq 0$, for $i \neq j$, and $\lambda_{i i}=-\sum_{j=1, j \neq i}^{\sigma} \lambda_{i j}$. We use $A_{i}, B_{i}$, and $H_{i}$ to denote the matrices of (1) when $\theta(t)=i \in \mathscr{S}$.

Our aim in this brief is to design a control rule in the linear state-feedback format with no mode observation

$$
u(t)=G x(t) \quad \forall t \geq 0 .
$$

Note that $G$ is a $(s \times r)$-D matrix independent of the Markov chain $\theta(t)$.

The control in (2) goes in the opposite direction of most of the results in the literature, which assume that the controller has full access to the Markovian mode [3]-[7].

As observed in [16], the assumption that the controller measures $\theta(t)$ signifies that the controller knows exactly and instantaneously what mode is active at $t>0$, which is unrealistic for many real-time processes. This brief confirms this statement.

Here, we present a real-time application for which the controller is blind with respect to $\theta(t)$-the application is for a dc-dc buck converter, a device largely used by the electronic industry. To control the buck converter in practice, we used the strategy in (2).

To design the gain matrix $G$ as in (2), we consider the long-run average cost

$$
J(G)=\limsup _{T \rightarrow \infty} \frac{1}{T} \int_{t=0}^{T} E\left[x(t)^{\prime} Q_{\theta(t)} x(t)+u(t)^{\prime} R_{\theta(t)} u(t)\right]
$$

where $E[\cdot]$ denotes the mathematical expectation conditioned to $\mathcal{F}_{0}=\left(x_{0}, \theta_{0}\right)$, and $Q_{i} \geq 0$ and $R_{i}>0, i \in \mathscr{S}$, are the given symmetric matrices.

The control problem we are interested in solving is

$$
\min _{G} J(G) \text { s.t. (1)-(3). }
$$

The problem in (4) is of difficult solution. To the best of the authors' knowledge, there is no global optimization method that yields the optimal solution for the problem posed in (4). As an attempt to overcome such a difficulty, we propose an algorithm for computing a candidate for the optimal solution in (4). This candidate satisfies a necessary optimality condition. Besides, this candidate is computed by a globally convergent algorithm. These findings represent the main theoretical contribution of this brief. 
The usefulness of our algorithm was checked in practice. A laboratory test bed was assembled with a power source that supplied a dc-dc buck converter connected with a load. Both the power source and load were programmed to suffer random changes on their nominal values according to a continuoustime homogeneous Markov chain.

The experimental setup has practical interest since random fluctuations in the nominal value of loads and sources are quite common in practice. The laboratory test bed was then constructed to reproduce such a random phenomenon, arranged to cope with the Markovian setup. In the laboratory, our algorithm generated the control strategy that was implemented in practice in the $\mathrm{dc}-\mathrm{dc}$ buck converter-the corresponding experimental data support the benefits of our algorithm.

This brief is organized as follows. Section II quotes the basic notation and presents the main result. Section III presents the real-time application for the dc-dc buck converter. Finally, Section IV presents some concluding remarks.

\section{Notation, Definitions, AND Basic Results}

The linear space made up by all $r \times s(r \times r)$ real matrices is denoted by $\mathcal{M}^{r, s}\left(\mathcal{M}^{r}\right)$. Let $\mathscr{S}:=\{1, \ldots, \sigma\}$ be an index set, and let $\mathbb{M}^{r, s}$ denote the linear space formed by $\sigma$ matrices belonging to $\mathcal{M}^{r, s}$, i.e., $\mathbb{M}^{r, s}=\left\{U=\left(U_{1}, \ldots, U_{\sigma}\right)\right.$ : $U_{i} \in \mathcal{M}^{r, s}, i \in \mathscr{S}$. Take, in particular, $\mathbb{M}^{r} \equiv \mathbb{M}^{r, r}$. We employ the ordering $U>V(U \geq V)$ for elements of $\mathbb{M}^{r}$, meaning that $U_{i}-V_{i}$ is positive definite (semidefinite) for all $i \in \mathscr{S}$, and similarly for other mathematical relations. If $V \in \mathcal{M}^{r, s}$ and $U \in \mathbb{M}^{s, r}$, then the multiplication $V U$ results in $\left(V U_{1}, \ldots, V U_{\sigma}\right) \in \mathbb{M}^{r, r}$. The trace operator is denoted by $\operatorname{tr}\{\cdot\}$. For elements from $\mathbb{M}^{r, s}$, we define the inner product

$$
\langle U, V\rangle=\sum_{i=1}^{\sigma} \operatorname{tr}\left\{U_{i}^{\prime} V_{i}\right\} \quad \forall U, V \in \mathbb{M}^{r, s} .
$$

Let us define the conditional second moment matrix of the system state $x(t), t \geq 0$, as

$$
X_{i}(t)=\mathrm{E}\left[x(t) x(t)^{\prime} 11_{\{\theta(t)=i\}} \quad \forall i \in \mathscr{S} \forall t \geq 0\right.
$$

where $11_{\{\cdot\}}$ stands for the Dirac measure. It is known from the literature that the second moment matrix in (5) can be evaluated using the next set of differential equations [2, Prop. 5.3]

$$
\begin{aligned}
\dot{X}_{i}(t)= & \left(A_{i}+B_{i} G\right) X_{i}(t)+X_{i}(t)\left(A_{i}+B_{i} G\right)^{\prime} \\
& +\sum_{j=1}^{\sigma} \lambda_{j i} X_{j}(t)+p_{i}(t) H_{i} H_{i}^{\prime} \quad \forall i \in \mathscr{S} \forall t \geq 0
\end{aligned}
$$

with $X_{i}(0)=p_{i}(0) x_{0} x_{0}^{\prime}$ and $p_{i}(t)=\operatorname{Pr}(\theta(t)=i)$ for each $i \in \mathscr{S}$ and every $t \geq 0$. Interestingly, the boundedness of $X(t)$ as long as $t$ increases can be assured by stabilizing gains $G$, a concept introduced next.

Definition 1: We say the gain $G$ is mean square stabilizing if the corresponding autonomous system $\dot{z}(t)=\left(A_{\theta(t)}+\right.$ $\left.B_{\theta(t)} G\right) z(t)$ satisfies $\lim _{t \rightarrow \infty} \mathrm{E}\left[\|z(t)\|^{2}\right]=0$ for any initial condition $\left(z_{0}, \theta_{0}\right)$.

Consider the next assumption.

Assumption 2: $\{\theta(t)\}$ is an irreducible positive Harris recurrent Markov process.
The result in [2, Prop 5.4], quoted next, requires the condition in Assumption 2.

Proposition 3 ([2, Prop 5.4]): If $G$ is a mean-square stabilizing gain, then the limits

$$
X_{i}:=\lim _{t \rightarrow \infty} X_{i}(t), \quad i=1, \ldots, \sigma
$$

exist and do not depend on the initial condition $\left(x_{0}, \theta_{0}\right)$.

The result of Proposition 3, applied in (6), allows us to obtain an equivalent form of representing the average cost (3) (for a proof, see [21, Th. 1]).

Proposition 4: If $G$ is a mean-square stabilizing gain, then

$$
J(G)=\sum_{i=1}^{\sigma} \operatorname{tr}\left\{X_{i}\left(Q_{i}+G^{\prime} R_{i} G\right)\right\}
$$

where $X \in \mathbb{M}^{r}$ satisfies

$$
\begin{array}{r}
\left(A_{i}+B_{i} G\right) X_{i}+X_{i}\left(A_{i}+B_{i} G\right)^{\prime}+\sum_{j=1}^{\sigma} \lambda_{j i} X_{j}+p_{i} H_{i} H_{i}^{\prime}=0 \\
\forall i \in \mathscr{S} .
\end{array}
$$

Note that the stochastic control problem in (3) is converted into the next one

$$
\min _{G} J(G) \text { s.t. (7)-(8). }
$$

\section{A. Lagrangian Equation and Necessary Optimality Conditions}

To characterize a solution for the control problem (9), let $L \in \mathbb{M}^{r}$ be the solution of the equation

$$
\begin{aligned}
Q_{i}+ & G^{\prime} R_{i} G+L_{i}\left(A_{i}+B_{i} G\right)+\left(A_{i}+B_{i} G\right)^{\prime} L_{i} \\
& +\sum_{j=1}^{\sigma} \lambda_{i j} L_{j}=0 \quad \forall i \in \mathscr{S} .
\end{aligned}
$$

Assumption 5: The pairs $\left(A_{i}, Q_{i}^{1 / 2}\right), i=1, \ldots, \sigma$, are observable in the deterministic sense (see [22, Ch. 6]).

The next result is an immediate consequence of [23, Th. 29].

Proposition 6 [23, Th. 29]: Suppose that $L \geq 0$ represents a solution of (10) for some given $G$. Then $G$ is mean square stabilizing.

For some given $X, L \in \mathbb{M}^{r}$, consider the Lagrangian equation

$$
\begin{aligned}
& \mathscr{L}(G, X, L) \\
& =J(G)+\sum_{i=1}^{\sigma} \operatorname{tr}\left\{L _ { i } \left(\left(A_{i}+B_{i} G\right) X_{i}+X_{i}\left(A_{i}+B_{i} G\right)^{\prime}\right.\right. \\
& \left.\left.+\sum_{j=1}^{\sigma} \lambda_{j i} X_{j}+p_{i} H_{i} H_{i}^{\prime}\right)\right\} .
\end{aligned}
$$

The corresponding Lagrange equations are obtained by passing in (11) the partial derivative with respect to $X$ and $L$ and setting both expressions to zero; this procedure yields (8) and (10), respectively. In addition, passing in (11) the partial derivative with respect to $G$ produces

$$
\frac{\partial \mathscr{L}(G, X, L)}{\partial G}=\sum_{i=1}^{\sigma} 2\left(B_{i}^{\prime} L_{i} X_{i}+R_{i} G X_{i}\right) .
$$


A necessary optimality condition for the problem (9) is now presented.

Proposition 7: Let the gain $G^{*}$ be the optimal solution for the problem in (9), i.e., $G^{*}=\arg \min _{G} J(G)$. Then $G^{*}$ satisfies

$$
\left.\frac{\partial \mathscr{L}(G, X, L)}{\partial G}\right|_{G=G^{*}}=0
$$

where $X \in \mathbb{M}^{r}$ and $L \in \mathbb{M}^{r}$ satisfy (8) and (10), respectively.

Remark 8: A stationary point $G$ that satisfies simultaneously (8), (10), and (13) is difficult to compute because such equations are nonlinearly coupled in an intricate manner. Our algorithm aims to overcome such a drawback. That is to say, our algorithm is globally convergent and assuredly computes a gain $G$ satisfying (8), (10), and (13), as detailed next.

\section{B. Algorithm for the Control Problem}

Let us now consider the following algorithm.

Step 1: Set the iterations counter $k=0$. Pick an arbitrary mean-square stabilizing gain $G^{[0]}$.

Step 2: Solve both $X^{[k]}$ and $L^{[k]}$ according to (8) and (10), respectively. Compute the gradient

$$
\nabla^{[k]}:=\left.\frac{\partial \mathscr{L}\left(G, X^{[k]}, L^{[k]}\right)}{\partial G}\right|_{G=G^{[k]}}
$$

and verify whether $\left\|\nabla^{[k]}\right\|<\varepsilon$ holds true for some sufficiently small $\varepsilon>0$. In the positive case, stop the algorithm. Otherwise, compute the matrix

$$
S^{[k]}:=-\frac{1}{2} \nabla^{[k]}\left(\sum_{i=1}^{\sigma} X_{i}^{[k]}\right)^{-1}
$$

and go to Step 3).

Step 3: Find $a^{[k]}>0$ such that (Goldstein's condition [24, p. 118])

$$
\begin{aligned}
-\rho a^{[k]}\left\langle\nabla^{[k]}, S^{[k]}\right\rangle & <J\left(G^{[k]}\right)-J\left(G^{[k]}+a^{[k]} S^{[k]}\right) \\
& <-(1-\rho) a^{[k]}\left\langle\nabla^{[k]}, S^{[k]}\right\rangle
\end{aligned}
$$

where $0<\rho<0.5$ is a fixed parameter. Set $G^{[k+1]}:=G^{[k]}+a^{[k]} S^{[k]}$ and $k=k+1$, and return to the beginning of Step 2).

Remark 9: The variable $a^{[k]}>0$ in (16) can be computed using a backtracking algorithm (see [24, Sec. 6.3.2]). Other line-search strategies exist and can also be considered in such an evaluation, such as the Nelder-Mead method, pattern search method, and secant method [24], [25].

\section{Comments About the Algorithm of Section II-B}

The algorithm presented in Steps 1)-3) is inspired in quasiNewton methods, and as such, the algorithm generates a matrix sequence $\left\{S^{[k]}\right\}$ acting as a descent direction [24, Ch. 6]. To see this, note from (15) that

$$
\left\langle\nabla^{[k]}, S^{[k]}\right\rangle=-\frac{1}{2} \operatorname{tr}\left\{\nabla^{[k]^{\prime}} \nabla^{[k]}\left(\sum_{i=1}^{\sigma} X_{i}^{[k]}\right)^{-1}\right\}<0
$$

Since $\left\{J\left(G^{[k]}\right)\right\}$ is a monotonically nonincreasing sequence, and $L^{[k]}$ and $X^{[k]}$ exist, we can use Proposition 6 to obtain the next result.

Theorem 10: Each gain $G^{[k]}, k \geq 0$, generated by Steps 1)-3) is mean square stabilizing.

Other important conclusion follows by applying the result of [24, Th. 6.3.3, p. 121] in (17).

Theorem 11 (Global Convergence): The algorithm in Steps 1)-3) satisfies either $\nabla^{[k]}=0$ for some $k \geq 0$ or $\lim _{k \rightarrow \infty}\left\|\nabla^{[k]}\right\|=0$.

Remark 12: Theorem 11 is inconclusive with respect to the convergence of the gain sequence $\left\{G^{[k]}\right\}$. In applications, however, we can stop the algorithm of Steps 1)-3) at the $k_{0}$ th iteration when $\left\|\nabla^{\left[k_{0}\right]}\right\| \leq \varepsilon$, for some $\varepsilon>0$ sufficiently small.

\section{CONTROL APPLiCATION FOR A DC-DC BUCK CONVERTER}

A dc-dc buck converter is an electronic converter that supplies energy to a load, in a manner that the voltage across the load is less than or equal to the voltage from the sourcethis converter is indispensable in many industrial applications due to its high efficiency as well as its simplicity of control (see [26]-[30] for a brief account).

The voltage supplied to the dc-dc buck converter can suffer oscillations that many of them produced randomly (e.g., photovoltaic applications [31]). Such oscillations can generate undesired voltage oscillations in the load. Besides, when the nominal value of the load changes abruptly, a voltage overshoot appears over the load terminals.

That oscillations and overshoots on the load represent a risk of damage to the underlying device. To control the voltage applied in the load, thus mitigating the influence of voltage overshoots, we designed and implemented a continuous-time state feedback strategy in the dc-dc buck converter, as detailed next.

The aim of our experimental control project was to design a controller that mitigates the influence of voltage overshoots in the load, as well as keeping the voltage close to a certain reference. The control actuated to keep the load voltage regulated, even though Markov-driven disturbances affected the practical setup: the Markov chain sets the values of: 1) power source voltage and 2) nominal value of the load.

Experiments were performed in a laboratory to check the usefulness of our control strategy. The dc-dc buck converter was supplied by a power source that was programmed to change its voltage according to the state of a continuous-time Markov chain; this state also set the nominal value of the load. The algorithm in Steps 1)-3) was then used to compute a gain $G$, which was implemented in practice in the controller of the dc-dc buck converter.

The experimental data suggested that our control approach were appropriate to achieve the aim of mitigating voltage overshoots, as illustrated in the sequence. This finding sets the practical contribution of this brief.

\section{A. Modeling of the DC-DC Buck Converter}

The dc-dc buck converter is constructed with common electronic components, such as MOSFET $M$, diode $D$, 


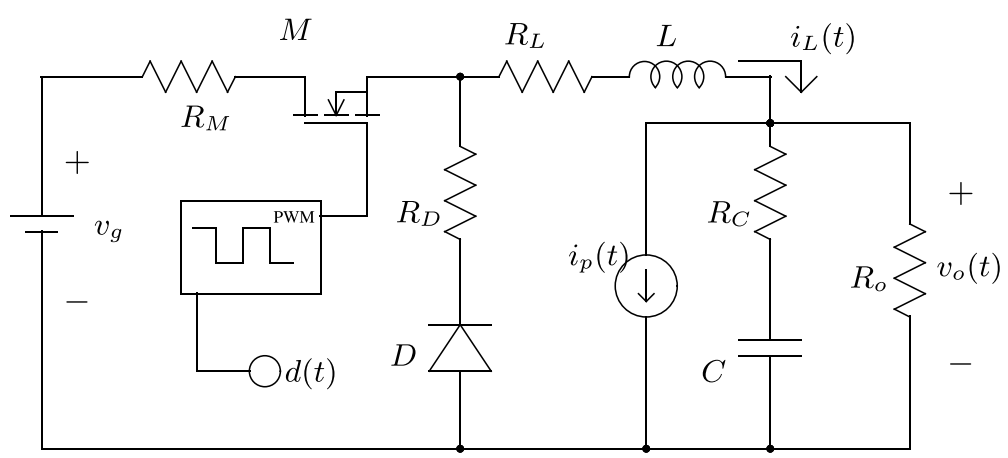

Fig. 1. Electronic circuit of the dc-dc buck converter. The main components are the inductor $L$, MOSFET $M$, diode $D$, capacitor $C$, and load $R_{O}$.

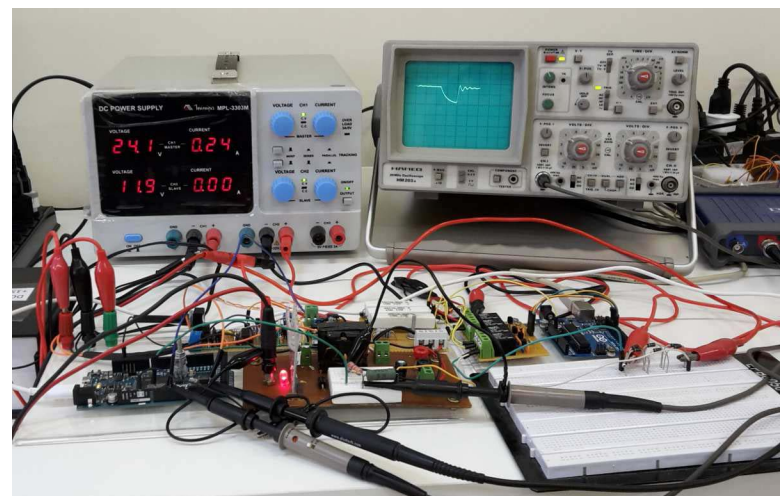

Fig. 2. Laboratory test bed. The picture shows the circuits and devices used in the experiments described in Section III.

inductor $L$, capacitor $C$, resistance $R_{O}$, and power supply $v_{g}$; these components are assembled as in the scheme shown in Fig. 1. In Fig. 1, the elements $R_{M}, R_{D}, R_{L}$, and $R_{C}$ stand for internal resistances of the corresponding components. The parasitic current is accounted in the variable $i_{p}(t)$. Both $v_{o}(t)$ and $i_{L}(t)$ are measured by sensors-they characterize the system state, as discussed next.

The MOSFET $M$ works under a pulsewidth modulation (PWM) signal, which keeps the MOSFET ON or OFF. The part of the time in which the MOSFET remains ON sets the duty cycle; the duty cycle is denoted here in its normalized value, i.e., $0 \leq d(t) \leq 1$, defining the input variable of the circuit (Fig. 1).

For simplicity, we neglect the resistances $R_{C}, R_{M}$, and $R_{D}$ in the circuit; the model of the dc-dc buck converter reads as (with $x(t) \equiv\left[v_{o}(t) i_{L}(t)\right]^{\prime}$; see Appendix A)

$$
\dot{x}(t)=\left[\begin{array}{cc}
-\frac{1}{R_{o} C} & \frac{1}{C} \\
-\frac{1}{L} & -\frac{R_{L}}{L}
\end{array}\right] x(t)+\left[\begin{array}{c}
0 \\
\frac{v_{g}}{L}
\end{array}\right] d(t)+\left[\begin{array}{c}
-\frac{i_{p}(t)}{C} \\
0
\end{array}\right] .
$$

Note that the duty cycle $d(t)$ acts as the variable input of the system (18).

\section{B. Laboratory and Experiments}

The circuit of Fig. 1 was assembled in a laboratory test bed (see Fig. 2). The laboratory was built up to check in practice
TABLE I

Markovian Modes of Operation OF THE DC-DC BUCK CONVERTER

\begin{tabular}{ccccccc}
\hline Components & $i=1$ & $i=2$ & $i=3$ & $i=4$ & $i=5$ & $i=6$ \\
$R_{o}$ & 30 & 20 & 10 & 30 & 20 & 10 \\
$v_{g}$ & 24.1 & 24.1 & 24.1 & 22 & 22 & 22 \\
\hline
\end{tabular}

the behavior of a dc-dc buck converter when both load and power supply are driven by a Markov chain. For this purpose, the laboratory was configured so as to change the value of the load $R_{o}$, according to a Markov chain, for next three values: 30, 20, and $10 \Omega$. Besides, the Markovian chain triggered the power supply to provide either 24.1 or $22 \mathrm{~V}$. Thus, the changes among the resistances and voltages followed the jumps of a six-mode Markov chain (Table I).

The Markov chain was implemented in an Arduino Uno, a microcontroller board; this board triggered relays that set values for the load and power supply. A digital oscilloscope, the model PicoScope-3404A, was used to measure and record the experimental data.

An analog-to-PWM driver was implemented in the laboratory to generate the input signal $d(t)$ in practice. The analog-to-PWM driver, based on the chip SG3524, had an analog input signal $u(t)$ ranging from 0 to $4 \mathrm{~V}$ and it had output with proportional duty cycle from 0 to $95 \%$. This proportional relationship allowed us to substitute $d(t)$ by $u(t)$ in (18).

Since both the load $R_{O}$ and the power supply $v_{g}$ were driven by a Markov chain, and taking into account that $R_{L}$ may change due to the switching action of the MOSFET (its internal ON and OFF resistances in series with the inductor), we obtain from (18) the next continuous-time MJLS

$$
\begin{aligned}
d x(t)= & {\left[\begin{array}{ll}
a_{11}^{(i)} & a_{12} \\
a_{21} & a_{22}^{(i)}
\end{array}\right] x(t) d t+\left[\begin{array}{c}
0 \\
b^{(i)}
\end{array}\right] u(t) d t } \\
& +\left[\begin{array}{cc}
h_{1} & 0 \\
0 & h_{2}
\end{array}\right] d w(t), \quad \theta(t)=i \in\{1, \ldots, 6\} \quad \forall t \geq 0
\end{aligned}
$$

with $\theta_{0}=1$ and $x_{0}=\left[\begin{array}{ll}0 & 0\end{array}\right]^{\prime}$, where the parameter values of (19) were identified through the next procedure (Table II).

In the laboratory, step inputs of distinct amplitudes were applied in $u(t)$ for every $i=1, \ldots, 6$, and the corresponding data for $v_{o}(t)$ and $i_{L}(t)$ were carefully recorded and compared 
TABLE II

Parameter Values of the Continuous-Time Markov Jump Linear System That Models the DC-DC Buck Converter

\begin{tabular}{|c|c|c|c|c|c|c|}
\hline Value $\left(\times 10^{3}\right)$ & $i=1$ & $i=2$ & $i=3$ & $i=4$ & $i=5$ & $i=6$ \\
\hline$a_{11}^{(i)}$ & -0.5012 & -0.7251 & -1.1484 & -0.6909 & -0.9466 & -1.4933 \\
\hline$a_{22}^{(i)}$ & -1.3292 & -1.1336 & -1.5964 & -1.0777 & -0.8985 & -0.9779 \\
\hline$b^{(i)}$ & 4.7166 & 4.6223 & 4.9239 & 3.6579 & 3.8118 & 4.0517 \\
\hline & $a_{12}=1.997$ & $a_{21}=-3.994$ & $h_{1}=1$ & $h_{2}=5$ & & \\
\hline
\end{tabular}

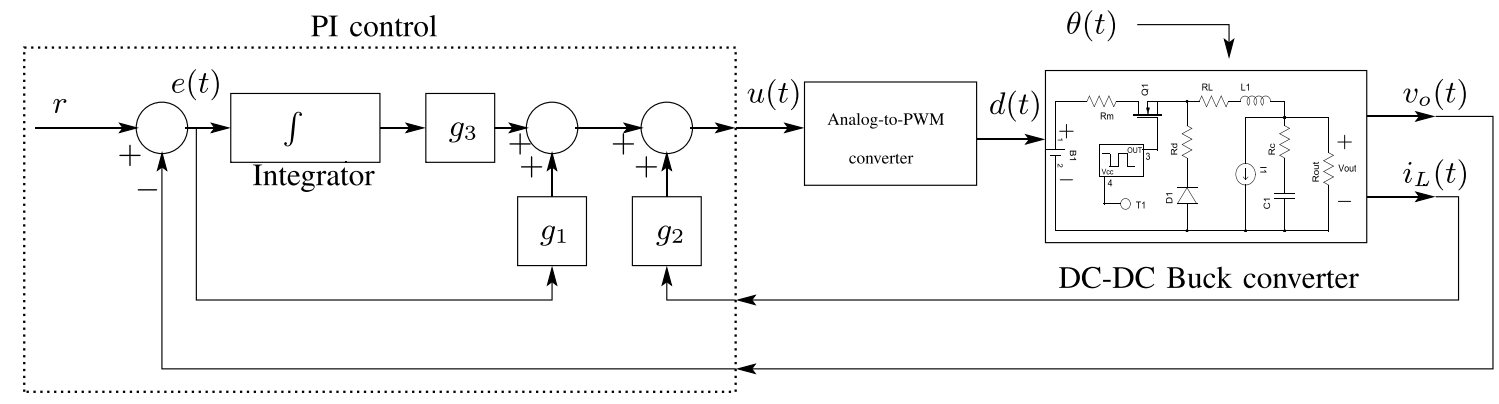

Fig. 3. State-feedback scheme used in practice to control a dc-dc buck converter driven by a continuous-time Markov chain.
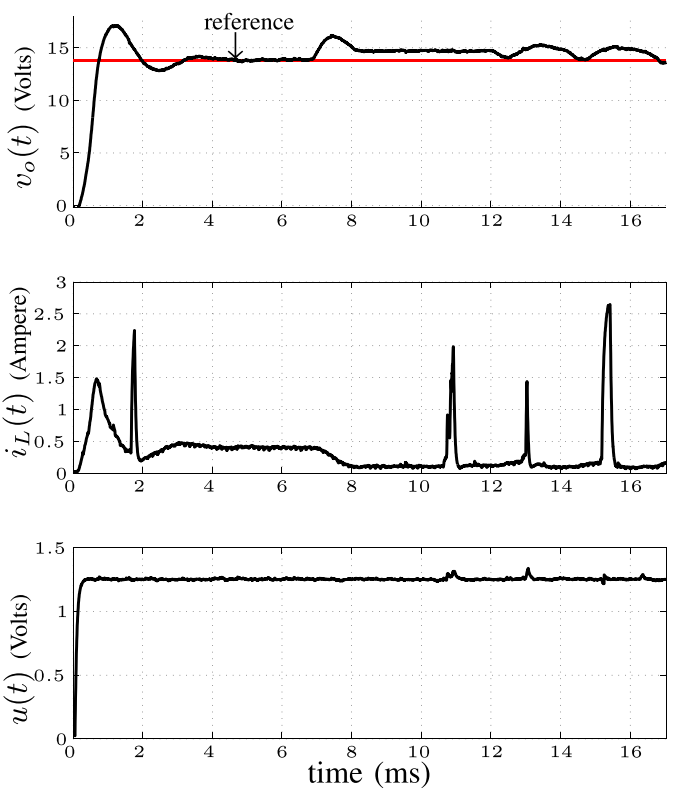
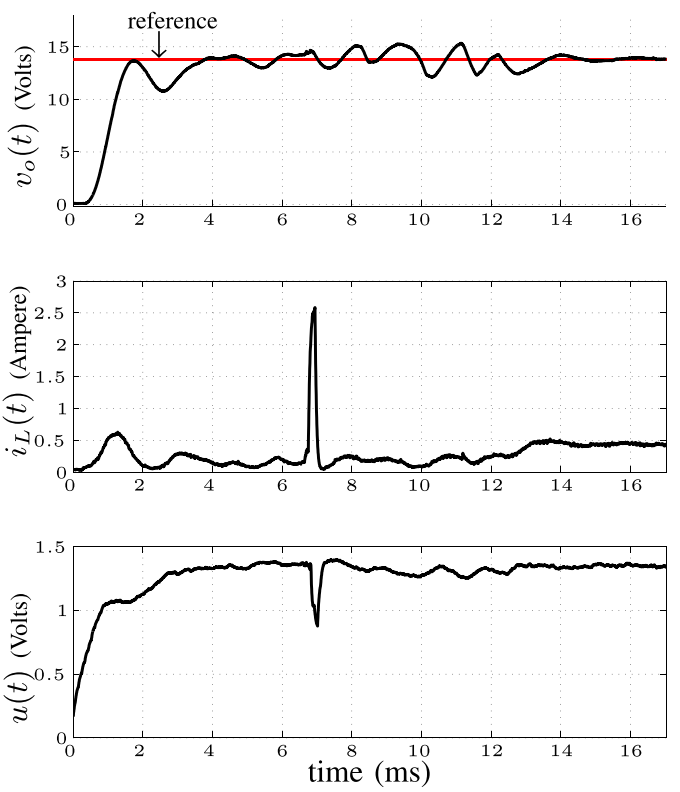

Fig. 4. Experimental data for some realization of the dc-dc buck converter. Open-loop response (left). Closed-loop response (right).

with the simulated counterpart from (19) with $w(t) \equiv 0$. The difference between the square of practical data and simulated data produced an error, and by minimizing the integral of such an error, we obtained the values shown in Table II. The values of $h_{1}$ and $h_{2}$ were obtained via an analysis of the statistical dispersion of the practical data.

\section{Experimental Results: Controlled DC-DC Buck Converter With Markov Jumps}

This section illustrates the usefulness of Theorem 11.

The proportional-integrative (PI) control strategy has been used in the control of processes subject to Markovian jumps [16], [17], showing promising results therein. For this reason, we decided to apply the PI strategy here to control the dc-dc buck converter, as shown in the scheme of Fig. 3. As can be seen, the proposed control action is

$$
\begin{aligned}
& u(t)=g_{1} e(t)+g_{2} i_{L}(t)+g_{3} \int_{0}^{t} e(\tau) d \tau \\
& e(t)=r-v_{o}(t) \quad \forall t \geq 0
\end{aligned}
$$

where $r$ denotes a fixed set-point signal.

The control objective of the practical experiment was to assure that the statistical mean value of $e(t)$ tends to zero as $t$ tends to infinity.

Let $q(t)$ be the integral of the error, i.e., $q(t):=$ $\int_{\tau=0}^{t} e(\tau) d \tau$, so that $\dot{q}(t) \equiv e(t)$. Now, take the system state as $x(t) \equiv\left[\begin{array}{lll}v_{o}(t) & i_{L}(t) & q(t)\end{array}\right]^{\prime}$. With $G=\left[\begin{array}{lll}-g_{1} & g_{2} & g_{3}\end{array}\right]$, it follows from (20) that the control equals $u(t)=G x(t)+g_{1} r$, 


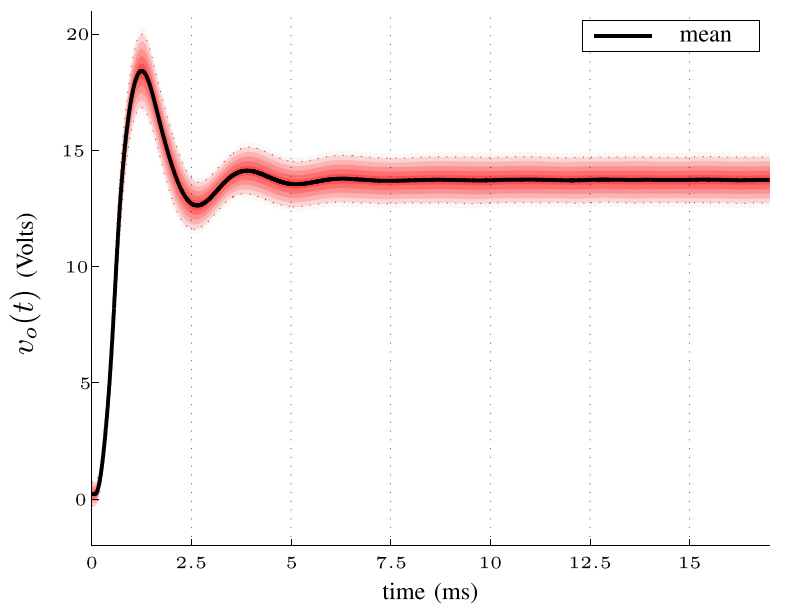

(a)

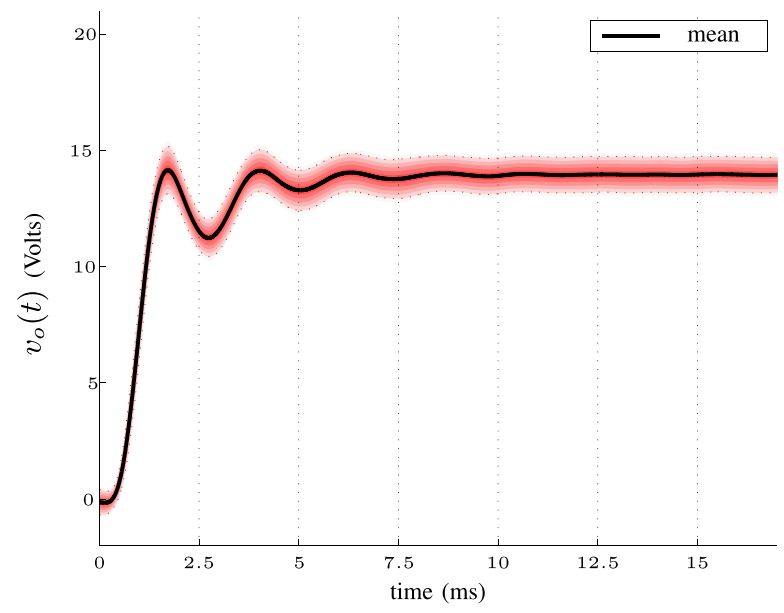

(b)

Fig. 5. Voltage in the load of a dc-dc buck converter. The shading area (in red) represents the statistical dispersion around the mean (in black) of the voltage measured for four thousand distinct realizations. The curves indicate that the result of Theorem 11 generated an improved response, i.e., lower overshoots and lower dispersion of voltage on the load. (a) Open loop. (b) Closed loop.

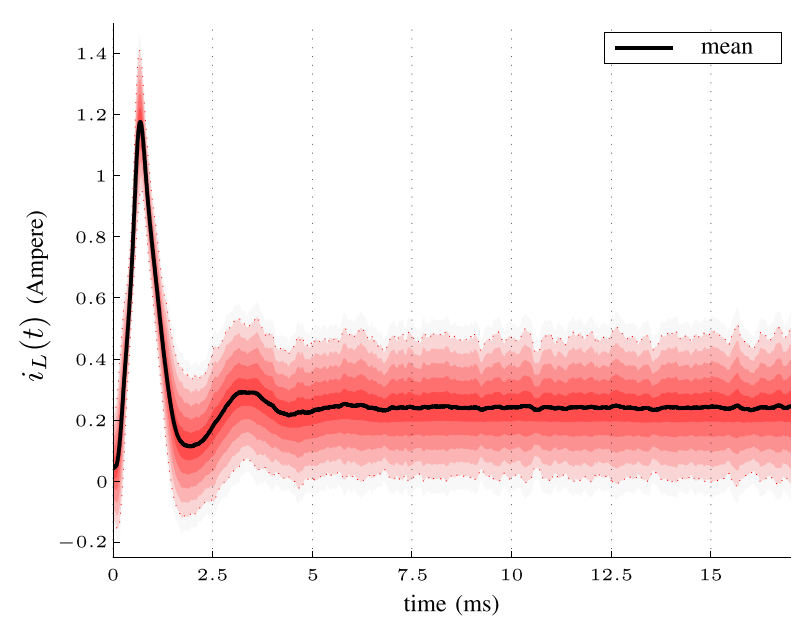

(a)

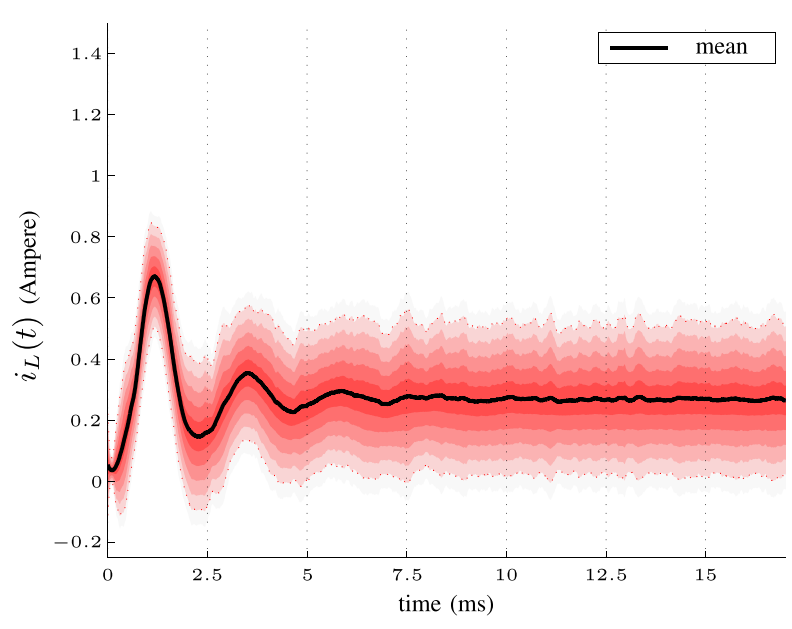

(b)

Fig. 6. Electric current flowing through the inductor of a dc-dc buck converter. The shading area (in red) represents the statistical dispersion around the mean (in black) of the electric current measured for four thousand distinct realizations. (a) Open loop. (b) Closed loop.

so that (19) implies in:

$$
\begin{array}{r}
d x(t)=\left(\left[\begin{array}{ccc}
a_{11}^{(i)} & a_{12} & 0 \\
a_{21} & a_{22}^{(i)} & 0 \\
-1 & 0 & 0
\end{array}\right]+\left[\begin{array}{c}
0 \\
b^{(i)} \\
0
\end{array}\right] G\right) x(t) d t \\
+\left[\begin{array}{c}
0 \\
b^{(i)} g_{1} r \\
r
\end{array}\right] d t+\operatorname{diag}(1,5,0.1) d w(t) \\
\theta(t)=i \in\{1, \ldots, 6\} .
\end{array}
$$

Steps 1)-3) now play a key role for setting the gain $G=\left[\begin{array}{lll}-g_{1} & g_{2} & g_{3}\end{array}\right]$ in (21). Indeed, we computed $G$ from the algorithm of Steps 1$)-3)$ with $R_{i} \equiv 1, Q_{i} \equiv \operatorname{diag}(1,0.1,1)$. The converge criteria was $\varepsilon=10^{-4}$. The transition rate matrix $\Lambda$ is presented in Appendix $\mathrm{B}$-the chosen value for $\Lambda$ reflects our desire to produce in the experiments many jumps in a short period of time, although respecting the relays'
TABLE III

Control Design for a DC-DC Buck Converter: As Assured IN THEOREM 11, THE ITERATIVE VALues CONVERGE

\begin{tabular}{cll}
\hline Iter. $k$ & $G^{[k]}$ & $\left\|\nabla^{[k]}\right\|\left(\times 10^{-4}\right)$ \\
\hline 0 & {$\left[\begin{array}{llll}0.0287 & 0.316 & 0.000077\end{array}\right]$} & $6.01 \times 10^{10}$ \\
1 & {$\left[\begin{array}{lll}0.0287 & 0.316 & 0.00095\end{array}\right]$} & $9.36 \times 10^{7}$ \\
2 & {$\left[\begin{array}{lll}0.0287 & 0.316 & 0.01\end{array}\right]$} & $61.62 \times 10^{4}$ \\
3 & {$\left[\begin{array}{lll}0.0277 & 0.31 & 0.12\end{array}\right]$} & $5.6 \times 10^{3}$ \\
4 & {$\left[\begin{array}{lll}-0.18 & -1.019 & 2.45\end{array}\right]$} & $1.04 \times 10^{3}$ \\
5 & {$\left[\begin{array}{lll}0.208 & -0.501 & 1.55\end{array}\right]$} & 21.7 \\
6 & {$\left[\begin{array}{lll}0.2465 & -0.3656 & 1.204\end{array}\right]$} & 8.85 \\
7 & {$\left[\begin{array}{lll}0.2466 & -0.3653 & 1.2017\end{array}\right]$} & 0.76 \\
\hline
\end{tabular}

physical restrictions. In particular for Step 3), we used the backtracking algorithm (see Remark 9 in connection).

The results from Steps 1)-3) are presented in Table III. As can be seen, the convergence in Table III confirms the result of Theorem 11. Besides, as a byproduct of Theorem 11, 


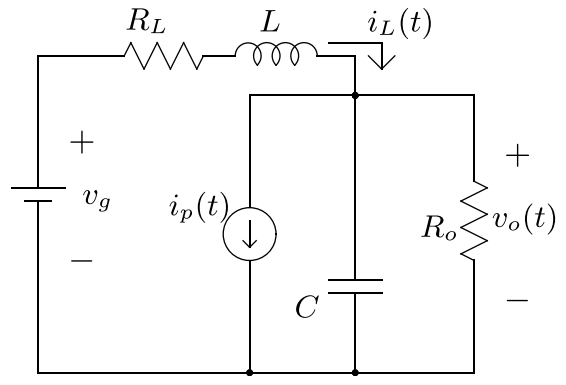

(a)

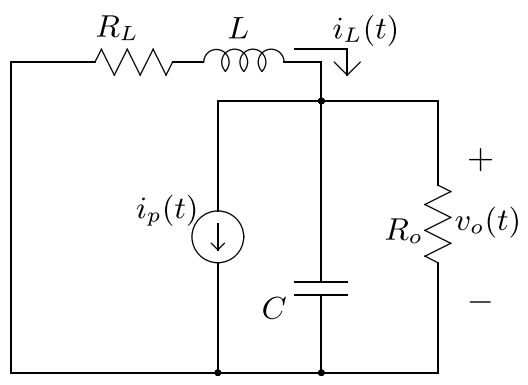

(b)

Fig. 7. Circuit of the dc-dc buck converter when the MOSFET is either ON or OFF. (a) MOSFET is ON. (b) MOSFET is OFF.

the gain $G=[-0.2466-0.36531 .2017]$ is not only mean square stabilizing but also candidate for the optimal solution of the control problem in (4).

The experiments were carried out to check the behavior of the dc-dc buck converter subject to Markov-driven operation modes (see Table I). The laboratory accounted for experiments with open loop (no control) and closed loop (control).

Regarding the closed-loop case, the control gain $G=$ [ $-0.2466-0.36531 .2017]$ was introduced in the PI control (Fig. 3), which was evaluated in the laboratory test bed with reference fixed at $r=13.85 \mathrm{~V}$. An Arduino Due with sampling time of around $90 \mathrm{~ms}$ performed the PI control in practice.

Four thousand realizations were conducted for the two cases, i.e., open loop and closed loop.

Fig. 4 shows the data for some realization. Despite the $\mathrm{dc}-\mathrm{dc}$ buck converter had been changing abruptly its operation mode, as demonstrated in the inductor current, the closed-loop response of the converter tended to follow the reference.

Fig. 5 summarizes the experimental data. The experimental data indicated that the PI control with $G$ given by Steps 1)-3) improved the overall dc-dc buck converter's response. Actually, when working under our control strategy, the converter presented lower overshoots as well as lower voltage dispersion on the load (see Fig. 5). Consequently, the proposed closed loop diminished voltage variations over the load, showing the potential of our approach for applications.

Finally, lower voltage overshoots in the load guarantee lower current overshoots in the inductance, as shown in Fig. 6. These findings are a positive indication toward the practical benefits of our control strategy.

\section{CONCLUSiON}

Our findings reveal two points.

First, we present an algorithm that computes a candidate for solving a difficult control problem-the long-run average cost control problem of MJLSs with inaccessible modes.
Second, we show that our algorithm has potential for applications - the algorithm was used in practice to design a controller for a dc-dc buck converter subject to Markov-driven modes.

A laboratory test bed was assembled to check the behavior of the dc-dc buck converter under distinct modes of operation. As long as this converter had been working, a Markov chain set the activated mode; every mode represented distinct values for load and power supply (Table I). In this scenario, the experiments were carried out for two cases: open loop (no control) and closed loop (control). The experimental data suggested that our approach have potential for real-time applications.

\section{APPENDIX A}

In this Appendix, we derive the model of the dc-dc buck converter presented in (18) (see [27], [29], [30] for further details). Recall the circuit of Fig. 1. When the MOSFET is either ON or OFF, we retrieve the circuits shown in Fig. 7.

Consider the system state as $x(t) \equiv\left[v_{o}(t) i_{L}(t)\right]^{\prime}$. When the MOSFET is ON, we can derive from Fig. 7(a) that

$$
\dot{x}(t)=\left[\begin{array}{cc}
-\frac{1}{R_{o} C} & \frac{1}{C} \\
-\frac{1}{L} & -\frac{R_{L}}{L}
\end{array}\right] x(t)+\left[\begin{array}{c}
0 \\
\frac{v_{g}}{L}
\end{array}\right]+\left[\begin{array}{c}
-\frac{i_{p}(t)}{C} \\
0
\end{array}\right]
$$

but when the MOSFET is OFF, we have from Fig. 7(b) that

$$
\dot{x}(t)=\left[\begin{array}{cc}
-\frac{1}{R_{o} C} & \frac{1}{C} \\
-\frac{1}{L} & -\frac{R_{L}}{L}
\end{array}\right] x(t)+\left[\begin{array}{c}
-\frac{i_{p}(t)}{C} \\
0
\end{array}\right] .
$$

Multiplying (22) by $d(t)$ and (23) by $(1-d(t))$, and summing up both, we obtain the model of the dc-dc buck converter in (18).

\section{APPENDIX B}

The rate transition matrix is presented as the equation shown at the bottom of this page.

$$
\Lambda=\left[\begin{array}{cccccc}
-96.3100 & 96.3100 & 0 & 0 & 0 & 0 \\
23.2472 & -99.6310 & 46.4945 & 0 & 16.6052 & 13.2841 \\
9.9631 & 66.4207 & -109.5941 & 16.6052 & 16.6052 & 0 \\
3.3210 & 9.9631 & 13.2841 & -66.4207 & 33.2103 & 6.6421 \\
0 & 16.6052 & 26.5683 & 249.0776 & -312.1772 & 19.9262 \\
0 & 0 & 19.9262 & 19.9262 & 19.9262 & -59.7786
\end{array}\right]
$$




\section{REFERENCES}

[1] O. L. V. Costa, M. D. Fragoso, and M. G. Todorov, ContinuousTime Markov Jump Linear Systems (Probability and Its Applications). New York, NY, USA: Springer-Verlag, 2013.

[2] M. D. Fragoso and O. L. V. Costa, "A unified approach for stochastic and mean square stability of continuous-time linear systems with Markovian jumping parameters and additive disturbances," SIAM J. Control Optim., vol. 44, no. 4, pp. 1165-1191, 2005.

[3] V. Dragan, T. Morozan, and A. Stoica, " $H^{2}$ optimal control for linear stochastic systems," Automatica, vol. 40, no. 7, pp. 1103-1113, 2004.

[4] E. F. Costa, A. N. Vargas, and J. B. R. do Val, "Quadratic costs and second moments of jump linear systems with general Markov chain," Math. Control, Signals, Syst., vol. 23, no. 1, pp. 141-157, 2011.

[5] A. N. Vargas, W. Furloni, and J. B. R. do Val, "Second moment constraints and the control problem of Markov jump linear systems," Numer. Linear Algebra Appl., vol. 20, no. 2, pp. 357-368, 2013.

[6] R. C. L. F. Oliveira, A. N. Vargas, J. B. R. do Val, and P. L. D. Peres, "Robust stability, $\mathscr{H}_{2}$ analysis and stabilisation of discrete-time Markov jump linear systems with uncertain probability matrix," Int. J. Control, vol. 82, no. 3, pp. 470-481, 2009.

[7] L. Wu, P. Shi, and H. Gao, "State estimation and sliding-mode control of Markovian jump singular systems," IEEE Trans. Autom. Control, vol. 55, no. 5, pp. 1213-1219, May 2010.

[8] L. Zhang, E.-K. Boukas, L. Baron, and H. R. Karimi, "Fault detection for discrete-time Markov jump linear systems with partially known transition probabilities," Int. J. Control, vol. 83, no. 8, pp. 1564-1572, 2010.

[9] L. Zhang and J. Lam, "Necessary and sufficient conditions for analysis and synthesis of Markov jump linear systems with incomplete transition descriptions," IEEE Trans. Autom. Control, vol. 55, no. 7, pp. 1695-1701, Jul. 2010.

[10] L. Zhang, " $H_{\infty}$ estimation for discrete-time piecewise homogeneous Markov jump linear systems," Automatica, vol. 45, no. 11, pp. 2570-2576, 2009.

[11] P. Bolzern, P. Colaneri, and G. De Nicolao, "Markov jump linear systems with switching transition rates: Mean square stability with dwell-time," Automatica, vol. 46, no. 6, pp. 1081-1088, 2010.

[12] H. Shen, J. H. Park, L. Zhang, and Z.-G. Wu, "Robust extended dissipative control for sampled-data Markov jump systems," Int. J. Control, vol. 87, no. 8, pp. 1549-1564, 2014.

[13] P. Shi and F. Li, "A survey on Markovian jump systems: Modeling and design," Int. J. Control, Autom., Syst., vol. 13, no. 1, pp. 1-16, Feb. 2015.

[14] F. Li, L. Wu, P. Shi, and C.-C. Lim, "State estimation and sliding mode control for semi-Markovian jump systems with mismatched uncertainties," Automatica, vol. 51, pp. 385-393, Jan. 2015.

[15] P. Shi, Y. Yin, F. Liu, and J. Zhang, "Robust control on saturated Markov jump systems with missing information," Inf. Sci., vol. 265, pp. 123-138, May 2014.
[16] A. N. Vargas, E. F. Costa, and J. B. R. do Val, "On the control of Markov jump linear systems with no mode observation: Application to a DC motor device," Int. J. Robust Nonlinear Control, vol. 23, no. 10, pp. 1136-1150, 2013.

[17] R. C. L. F. Oliveira, A. N. Vargas, J. B. R. do Val, and P. L. D. Peres, "Mode-independent $\mathcal{H}_{2}$-control of a DC motor modeled as a Markov jump linear system," IEEE Trans. Control Syst. Technol., vol. 22, no. 5, pp. 1915-1919, Sep. 2014.

[18] J. B. R. do Val and T. Başar, "Receding horizon control of jump linear systems and a macroeconomic policy problem," J. Econ. Dyn. Control, vol. 23, no. 8, pp. 1099-1131, 1999.

[19] J. P. Hespanha, P. Naghshtabrizi, and Y. Xu, "A survey of recent results in networked control systems," Proc. IEEE, vol. 95, no. 1, pp. 138-162, Jan. 2007.

[20] L. Zhang, H. Gao, and O. Kaynak, "Network-induced constraints in networked control systems-A survey," IEEE Trans. Ind. Informat., vol. 9, no. 1, pp. 403-416, Feb. 2013.

[21] O. L. V. Costa, J. B. R. do Val, and J. C. Geromel, "Continuous-time state-feedback $\mathrm{H}_{2}$-control of Markovian jump linear systems via convex analysis," Automatica, vol. 35, no. 2, pp. 259-268, 1999.

[22] C.-T. Chen, Linear System Theory and Design, 3rd ed. New York, NY, USA: Oxford Univ. Press, 1999.

[23] E. F. Costa and J. B. R. do Val, "On the observability and detectability of continuous-time Markov jump linear systems," SIAM J. Control Optim., vol. 41, no. 4, pp. 1295-1314, 2002.

[24] J. E. Dennis, Jr., and R. B. Schnabel, Numerical Methods for Unconstrained Optimization and Nonlinear Equations (Classics in Applied Mathematics), vol. 16. Philadelphia, PA, USA: SIAM, 1996.

[25] T. G. Kolda, R. M. Lewis, and V. Torczon, "Optimization by direct search: New perspectives on some classical and modern methods," SIAM Rev., vol. 45, no. 3, pp. 385-482, 2003.

[26] R. W. Erickson and D. Maksimović, Fundamentals of Power Electronics. New York, NY, USA: Springer, 2001.

[27] R. D. Middlebrook and S. Ćuk, "A general unified approach to modelling switching-converter power stages," Int. J. Electron., vol. 42, no. 6, pp. 521-550, 1977.

[28] B. Johansson, "DC-DC converters-Dynamic model design and experimental verification," Ph.D. dissertation, Dept. Ind. Elect. Eng. Autom., Lund Univ., Lund, Sweden, 2005. [Online]. Available: http://lup.lub.lu.se/record/24326

[29] L. Martínez-Salamero et al., "Analysis and design of a sliding-mode strategy for start-up control and voltage regulation in a buck converter," IET Power Electron., vol. 6, no. 1, pp. 52-59, Jan. 2013.

[30] G. S. Deaecto, J. C. Geromel, F. S. Garcia, and J. A. Pomilio, "Switched affine systems control design with application to DC-DC converters," IET Control Theory Appl., vol. 4, no. 7, pp. 1201-1210, 2010.

[31] M. A. G. de Brito, L. Galotto, L. P. Sampaio, G. E. de Azevedo e Melo, and C. A. Canesin, "Evaluation of the main MPPT techniques for photovoltaic applications," IEEE Trans. Ind. Electron., vol. 60, no. 3, pp. 1156-1167, Mar. 2013. 